\title{
EXPERIMENTAL INVESTIGATION OF METAMATERIAL FROM RINGER CONDUCTORS
}

\author{
Aleksandr Mironchev, Aleksandr Gorst* \\ National Research Tomsk State University, 634050, Tomsk, Russia
}

\begin{abstract}
In this work creation of structure from ring conductors having negative index of refraction is shown. Experimental confirmation and finding of index of refraction, namely its real and imaginary part taking into account the error, for single-layer, two-layer, three-layered and four-layer structure.
\end{abstract}

\section{Introduction}

At present, metamaterials attract the great interest of the scientific world with their unique properties, unattainable with the use of familiar materials. For antenna technology, metamaterials with its extraordinary properties provide additional possibilities: smaller dimensions, higher antenna gain and wider bandwidth.

In the last decade of the 20th century, intensive research was conducted in many countries on a wide range of issues related to the physics of complex electromagnetic media and artificial materials that are intended for use in modern radio and optical devices and devices. Impressive results in the field of theory were achieved, which made it possible to better understand the radiophysical principles of creating new electromagnetic materials, as well as to reveal potential possibilities and the range of achievable values of material parameters. Therefore, it is not by chance that the term "metamaterial" appeared on the verge of millennia.

\section{Experimental study}

Earlier authors of the real work presented results of numerical and computer modeling of the flat closed ring conductor and the possibility of creation metamaterial for radio frequency range [1].

For check and confirmation of the stated results it was decided to conduct pilot study.

Creations of the experimental model consists in selection of ring conductors, used at computer modeling. To measure distances between elements and layers of structure.

In quality swore for implementation of elemental annuluses, frothed polystyrene was used. Elemental annuluses have the sizes: Outer diameter is $8 \mathrm{~mm}$, inside diameter is $4 \mathrm{~mm}$, thickness is $1 \mathrm{~mm}$. Elements were located rather each other at distance with $1 \mathrm{~cm}$. Total

*Corresponding author: gorst93@gmail.com 
quantity of elements in one layer made 900 pieces. For the experimental model 4 layers with total quantity of elements of 3600 pieces were used (Figure1).

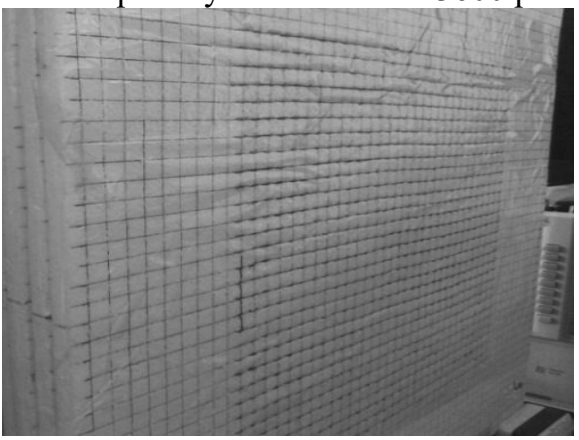

Fig.1. Experimental samples.

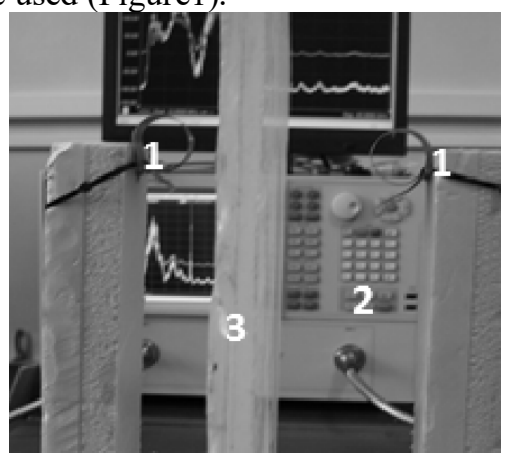

Fig. 2. Experimental installation:

1 - SShP the antenna, 2 - the complex plane analysis of chains, 3 - the studied sample.

Experiment carried out by the quasioptical method, based on measurement of parameters in free space.

For implementation of the method the installation presented in the Figure 2 is made. Installation includes the analyzer of chains PNA-L NetworkAnalyzer of the Agilent Technologies company and two transceiving antennas.

As transceiving antennas the combined "Snail" antennas representing constructive association of the electric and magnetic irradiator were used.

During the experiment five principal series of measurements were carried out:

1. The composite from ring conductors one layer, thickness is $1 \mathrm{~cm}$.

2. The composite from ring conductors two layers, thickness is $2 \mathrm{~cm}$.

3. Composite from ring conductors three layers, thickness of $3 \mathrm{~cm}$.

4. The composite from ring conductors four layers, thickness is $4 \mathrm{~cm}$.

5. Organic glass, thickness is $1.8 \mathrm{~cm}$.

It is necessary to make the normalization of the signal which passed through the studied sample on the signal received in its absence for finding of complex index of refraction. The regularization method according to by Tikhonov was used [2].

$$
S(f)=\frac{S_{2}(f) S_{1}^{*}(f)}{\left|S_{1}(f)\right|+\alpha},
$$

where $S_{1}$ - the signal in lack of the sample, $S_{2}$ - the signal which passed through the studied sample $\alpha$ - regularization coefficient.

As is well-known the Real part of index of refraction is determined by slope angle of the phase curve [2]. For this purpose on the chosen frequency interval it is carried out linear regression, presented in the form $y(x)=a+b x, a$ and $b$ reflection coefficients of the evaluated line. Using the ratio $\operatorname{Re}(n)=\frac{b \cdot c}{2 \pi \cdot d}$, where $c$ - the light speed, $d$ - thickness of the studied sample. Dependence of the phase and amplitude on frequency for the sample of organic glass $1.8 \mathrm{~cm}$ thick is given in the Figure 3.

The third straight line represented linear regression of the phase at frequencies from 2.5 $\mathrm{GHz}$ to $16 \mathrm{GHz}$. The schedule is constructed in half-logarithmic scale. On this interval of $\operatorname{Re}(n)=1.53$ that corresponds to tabular values. 


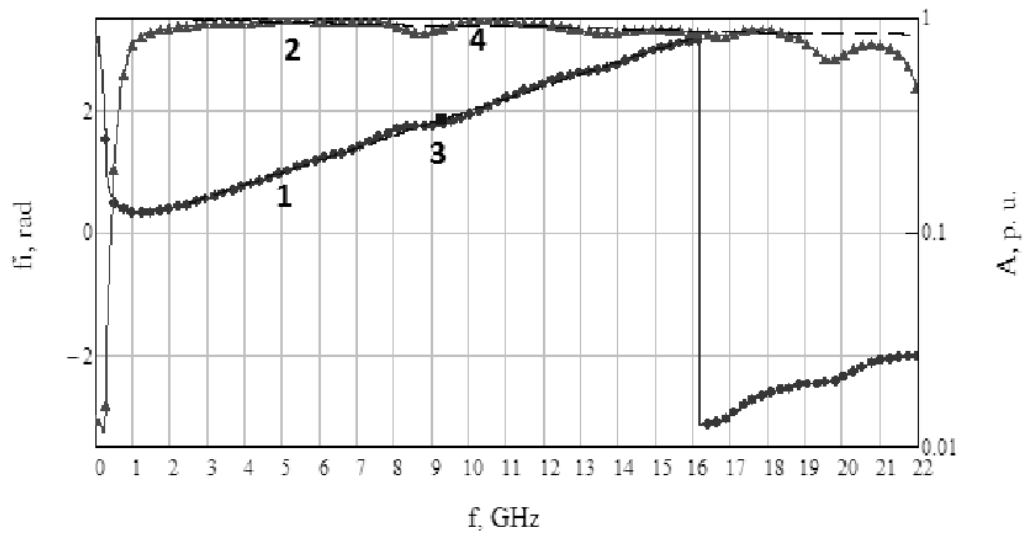

Fig. 3. Dependence of the phase (1) and amplitude (2) past of the signal from frequency, for the layer of organic glass $1.8 \mathrm{~cm}$ thick, linear regression of the phase (3), linear regression of amplitude (4).

Let's evaluate the imaginary part of complex index of refraction. As amplitude of last signal has the appearance: $A=\exp \left(-k n^{\prime \prime} d\right)$, where $k$ - wave number, $n^{\prime \prime}$ - imaginary part of index of refraction, $d$-thickness of the studied sample, $n^{\prime \prime}=\frac{\ln (1 / A) \cdot c}{2 \pi d}$.

The regression straight line shows the imaginary part of index of refraction, $\operatorname{Im}(n)=0.019$. By means of linear regression, perhaps to calculate the error of the received results.

The error of measurements was found with use of standard methods. Thus, we receive complex index of refraction for organic glass at frequencies from $2.5 \mathrm{GHz}$ to $16 \mathrm{GHz}$ of $n=1.530 \pm 0.001+\mathrm{i}(0.019 \pm 0.001)$.

Further with use of the same approach experimental measurements of samples of grids from elemental annuluses were taken. In the Figure 4 schedules of the experiment with the single-layer grid from elemental annuluses are submitted. Dependences of the phase (1) and amplitude (2) past of the signal are given in frequencies from $0.05 \mathrm{GHz}$ to $22 \mathrm{GHz}$. Lines (3) and (4) represented linear regression for the phase (3) and amplitude (4) respectively. Points noted mean values and confidential intervals.

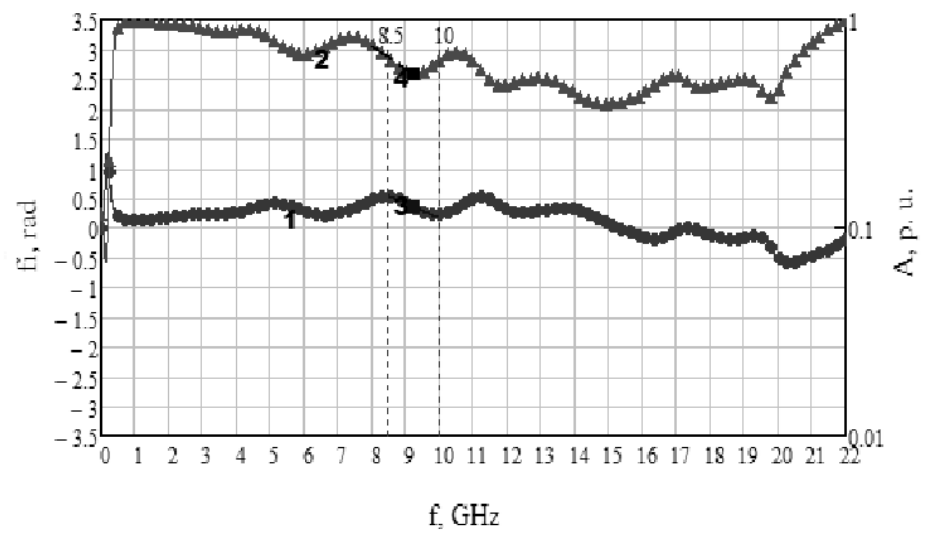

Fig. 4. Dependences amplitude (2) and phase (1) of last signal from frequency for one layer of rings $1 \mathrm{~cm}$ thick, linear regression of the phase (3), linear regression of amplitude (4). 
It is noticeable that on the interval from $8.5 \mathrm{GHz}$ to $10 \mathrm{GHz}$ at the phase reverse motion is observed.

At frequencies from $8.5 \mathrm{GHz}$ to $10 \mathrm{GHz}$, using the formulas given above, received complex index of refraction of $n=-0.21 \pm 0.02+\mathrm{i}(0.57 \pm 0.04)$. The turned-out result says that even the single-layer grid from the closed elemental annuluses is capable to give negative phase progression.

Let's consider results of the experiment made with two layers the Figure 5. It is possible to notice that, as well as in the previous case with one layer the phase of coefficient of passing has not linear character. Sites with the negative inclination of the phase curve which are characteristic of metamaterial are observed.

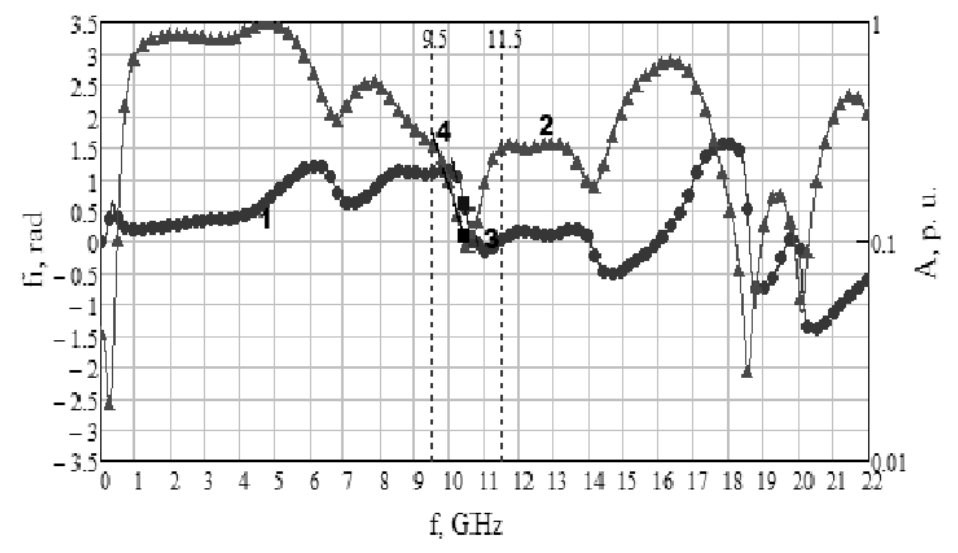

Fig. 5. Dependences of amplitude (2) and the phase (1) of last signal from frequency for two layers of rings $2 \mathrm{~cm}$ thick, linear regression of the phase (3), linear regression of amplitude (4).

In the Figure 5 dependences of amplitude and the phase of coefficient of passing are given in the interval from $9.5 \mathrm{GHz}$ to $11.5 \mathrm{GHz}$. Apparently from the drawing, at frequencies from 9.95-10.9 GHz phase progression reduction takes place. Complex index of refraction of $n=-3.843 \pm 0.13+\mathrm{i}(1.8 \pm 0.3)$. Such values of the real part of index of refraction testify to properties of metamaterial. But the imaginary part demonstrates rather strong attenuation at these frequencies. Such result can say that only one of the component the dielectric or magnetic component accepted negative value.

Further are given result of the experiment for three layers of rings $3 \mathrm{~cm}$ thick in the Figure 6. The behavior of curves is similar to the schedules provided for two layers with a general thickness of $2 \mathrm{~cm}$. Also frequency intervals on which phase attack has the negative course are observed. 


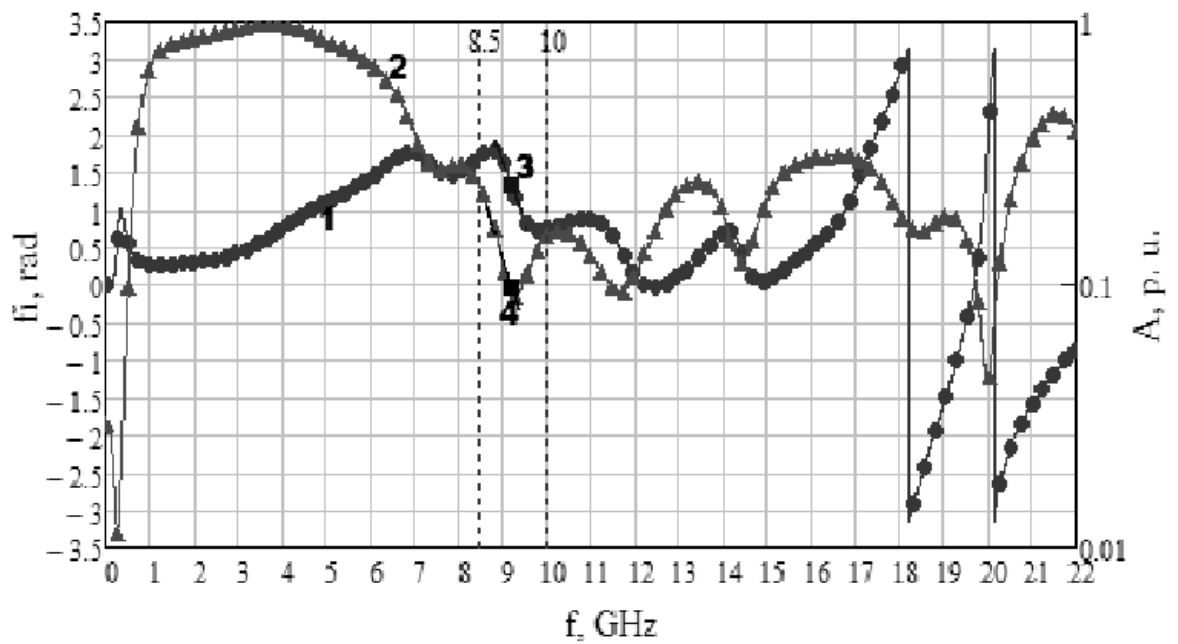

Fig. 6. Dependences of amplitude (2) and the phase (1) of last signal from frequency for three layers of rings $3 \mathrm{~cm}$ thick, linear regression of the phase (3), linear regression of amplitude (4).

In frequency band from $8.5 \mathrm{GHz}$ to $10 \mathrm{GHz}$ strong, but smooth recession of signal amplitude of the past through the three-layered grid is observed. At the same time it is possible to notice also decrease of phase attack with growth of frequency.

These observations speak about presence of properties of metamaterial at the studied sample. Complex index of refraction of $n=-1.432 \pm 0.05+\mathrm{i}(1.89 \pm 0.06)$. It is worth noticing that having increased quantity of layers, real part of index of refraction decreased on absolute value, and the imaginary part at the same time remained equal to value for two layers $(n=-3.84 \pm 0.13+\mathrm{i}(1.8 \pm 0.3))$.

Further the case with use of four layers of rings is considered.

The schedules showing dependence of amplitude and the phase of the signal which passed through the four-layer grid $4 \mathrm{~cm}$ thick are provided on the Figure 7. Dependence has similar character in relation to the previous cases. Not the linear nature of phase change of last signal is observed.

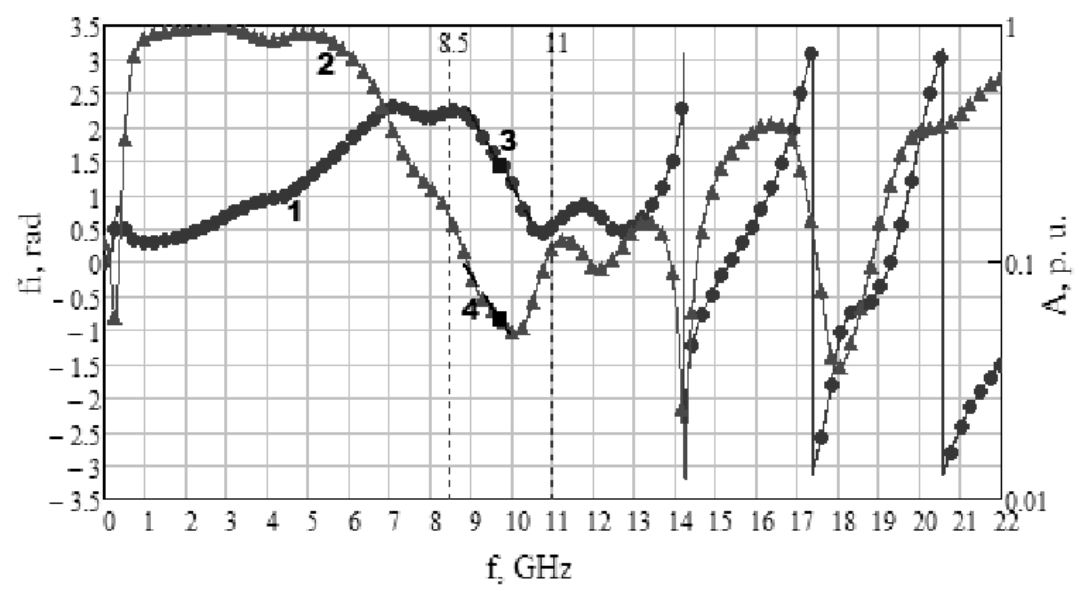

Fig. 7. Dependence of amplitude (2) and the phase (1) of last signal from frequency for four layers of rings $4 \mathrm{~cm}$ thick, linear regression of the phase (3), linear regression of amplitude (4). 
On the interval of frequencies from $8.5 \mathrm{GHz}$ to $11 \mathrm{GHz}$ there is the decrease, both phase attack, and amplitude. Amplitude of coefficient of passing has low values, and reaches the minimum at 0:05 relative units at the frequency of $10 \mathrm{GHz}$, at the same time phase attack continues to decrease to the frequency of equal $10.7 \mathrm{GHz}$. Complex index of refraction at these frequencies of $n=-0.27 \pm 0.01+\mathrm{i}(0.71 \pm 0.01)$.

\section{Conclusions}

By results of the experiment, it is possible to draw conclusions that grids from the elemental annuluses oriented definitely have properties of metamaterial, namely negative index of refraction. At increase in quantity of layers the real part of index of refraction decreases, at the same time its imaginary part increases. The minimum value of the real part $\operatorname{Re}(n)=-3.84 \pm 0.13$ at three layers. Further increase in quantity of layers, does not lead to reduction of the real part of complex index of refraction, and on the contrary increases it to values, single-layer space grid. It is worth noticing that reduction of the real part of index of refraction is connected with increase in its imaginary part. The submitted schedules show that reduction of phase attack happens only in the presence of attenuation in the sample. It occurs at the expense of the strong interferential fields arising in the near zone of the space grid. As energy of incident wave is spent for emergence of near-fields around each element. There are composite blocked interfering fields which interact with each other. Finally induced near-field does not give increase of the phase, and even compensates the phase attack acquired to the layer.

\section{References}

[1] A. Mironchev, A. Gorst, MATEC Web of Conferences 79, 1 (2016) doi: 10.1051/matecconf/20167901064

[2] G.A. Ponomarev, V.N. Ponomareva, V.P. Yakubov, Statistical Methods in Radiophysics: A Practical Course Using Dialog-Computing Complexes (Tomsk University Press, Tomsk, 1989) 